\title{
Finite Temperature Dynamical Correlations using the Microcanonical Ensemble and the Lanczos Algorithm
}

\author{
M.W. Long, ${ }^{1}$ P. Prelovšek, ${ }^{2}$ S. El Shawish, ${ }^{2}$ J. Karadamoglou, ${ }^{3}$ and X. Zotos ${ }^{3}$ \\ ${ }^{1}$ School of Physics, Birmingham University, Edgbaston, Birmingham, B15 2TT, England \\ ${ }^{2}$ Faculty of Mathematics and Physics, University of Ljubljana, \\ and J. Stefan Institute, 1000 Ljubljana, Slovenia \\ ${ }^{3}$ Institut Romand de Recherche Numérique en Physique des Matériaux (IRRMA), \\ EPFL, 1015 Lausanne, Switzerland
}

(Dated: October 29, 2018)

\begin{abstract}
We show how to generalise the zero temperature Lanczos method for calculating dynamical correlation functions to finite temperatures. The key is the microcanonical ensemble, which allows us to replace the involved canonical ensemble with a single appropriately chosen state; in the thermodynamic limit it provides the same physics as the canonical ensemble but with the evaluation of a single expectation value. We can employ the same system sizes as for zero temperature, but whereas the statistical fluctuations present in small systems are prohibitive, the spectra of the largest system sizes are surprisingly smooth. We investigate, as a test case, the spin conductivity of the spin- $1 / 2$ anisotropic Heisenberg model and in particular we present a comparison of spectra obtained by the canonical and microcanonical ensemble methods.
\end{abstract}

\section{INTRODUCTION}

The study of lattice quantum many body systems by the exact diagonalisation technique has proven popular at zero temperature $(T=0)$ where only the ground state is required, but it is of less use at finite temperature. The reason can be attributed to the different system sizes applicable, where for spin- $1 / 2$ the ground state can be found for up to $N \sim 30$ lattice sites, but the entire spectrum can readily be achieved for systems only up to $N \sim 16$. The intrinsic difficulties associated with applying the finitesize scaling method on such small systems severely limit finite temperature applications. At $T=0$ the continued fraction technique ${ }^{1,2}$ allows accurate calculations of dynamical correlations using only the machinery of the Lanczos algorithm, but unfortunately this technique has not been extended to finite $T$ where mostly full diagonalisation has been employed. As well as direct applications of the canonical ensemble, there is also a hybrid method which employs the canonical representation of dynamical correlation functions but uses a Lanczos basis to provide a set of orthogonal states ${ }^{3}$. This method allows access to larger systems than are accessible to full diagonalisation techniques but to smaller systems than the current proposal, which does not need details of all the states even in the Lanczos basis. In this article we extend the $T=0$ formalism to finite temperature by applying a microcanonical ensemble approach combined with the Lanczos method (MCLM) that provides smooth predictions for dynamical correlation functions at least at high temperatures.

The physical advance is to appreciate that in the thermodynamic limit the microcanonical ensemble is equivalent to the canonical one ${ }^{4.5}$, but for finite systems this is much easier to work with. The statistical fluctuations engendered by the microcanonical choice are a drawback for small systems but become controllable for large systems. In practice, as the finite $T$ calculations are much smoother, it is more natural to contemplate applying finite-size scaling than for their $T=0$ counterparts. The exponentially dense nature of a many particle spectrum in the bulk is the property that smoothes our calculations, a characteristic that is lost near the ground state where the spectrum is sparse.

Besides the computational interest of this proposal it is worth pointing out that, to our knowledge, no studies of the fundamental equivalence between the microcanonical and canonical ensemble for quantum dynamic correlations exist in the literature. Thus this work is a step in numerically exploring this basic postulate of nonequilibrium statistical mechanics; clearly, analytical studies are needed to clarify, for instance, the meaning of the microcanonical ensemble for a quantum system with dense spectrum as an average over a single quantum state (or a narrow window of states) and the finite size corrections inherent in this ensemble.

\section{THERMODYNAMIC ENSEMBLES}

In this section we will discuss how an arbitrary probability distribution can be used, under reasonable assumptions, to represent the canonical ensemble in the thermodynamic limit. The choice of a (unnormalized) distribution $\rho(\epsilon)$ in the thermodynamic limit can be examined by considering its Laplace transform,

$$
\begin{array}{r}
e^{f(\tau)}=\int_{0}^{\infty} d \epsilon e^{\epsilon \tau} \rho(\epsilon), \quad \Re \tau<0 \\
\rho(\epsilon)=\int_{-i \infty-\eta}^{+i \infty-\eta} \frac{d \tau}{2 \pi i} e^{-\tau \epsilon+f(\tau)},
\end{array}
$$

where $f(\tau)$ controls the properties of a distribution designated by $\rho(\epsilon)$. As examples, the following choices of 
$f(\tau)$ lead to,

$$
e^{f(\tau)}=\frac{1}{\beta-\tau} \mapsto \rho(\epsilon)=e^{-\beta \epsilon}
$$

the canonical ensemble,

$$
e^{f(\tau)}=e^{\lambda \tau} \mapsto \rho(\epsilon)=\delta(\lambda-\epsilon)
$$

the microcanonical ensemble at energy $\lambda$,

$$
e^{f(\tau)}=e^{\lambda \tau+\sigma^{2}(\tau-\beta)^{2} / 2} \mapsto \rho(\epsilon)=e^{-(\epsilon-\lambda)^{2} / 2 \sigma^{2}}
$$

the "Gaussian" ensemble at energy $\lambda$ and width $\sigma$.

If we examine the partition function $Z_{\rho}$, then,

$$
Z_{\rho}=\operatorname{Tr} \rho(\epsilon)=\int_{+i \infty}^{-i \infty} \frac{d \tau}{2 \pi i} e^{f(\tau)+\ln Z(\tau)}
$$

where $Z(\tau)$ is the canonical partition function. The physical idea behind the thermodynamic limit is that the partition function becomes immensely sharp when considered as a function of state space and becomes dominated by the large number of states with the correct thermodynamics; in practice fluctuating quantities can be replaced by their thermodynamic average with negligible error. Mathematically, an integral such as (5) may be approximated in the asymptotic thermodynamic limit using the idea of 'steepest descents' with negligible error,

$$
Z_{\rho} \propto e^{f\left(\beta^{*}\right)} Z\left(\beta^{*}\right)
$$

Here $\beta^{*}$ is chosen so that,

$$
\frac{\partial f}{\partial \tau}\left(\beta^{*}\right)=-\frac{1}{Z} \frac{\partial Z}{\partial \tau}\left(\beta^{*}\right)=\langle H\rangle
$$

and the average energy at the desired temperature is crucial. For the particular case of the microcanonical distribution $\lambda=\langle H\rangle$ and we need to employ states whose energy is the thermodynamic average as one might naively guess. Provided that $f(\tau)$ has only a weak dependence on the system parameters, then the two partition functions are essentially equivalent. It is also clear that provided $f(\tau)$ has the required properties that the 'steepest descents' is a good approximation, then any appropriate ensemble will provide the thermodynamic limit, for a particular temperature. It is quite natural to employ $f(\tau)=\lambda \tau+F(\tau)$ where $\lambda$ is extrinsic and $F(\tau)$ is intrinsic, in order to limit towards the microcanonical ensemble.

\section{DYNAMICAL CORRELATIONS IN THE MICROCANONICAL AND CANONICAL ENSEMBLE}

The usually studied quantities of direct physical interest are the dynamic structure function,

$$
S(\mathbf{q}, \omega)=\int_{-\infty}^{+\infty} d t e^{i \omega t}\left\langle X_{\mathbf{q}}(t) X_{-\mathbf{q}}(0)\right\rangle,
$$

and dynamic susceptibility,

$$
\chi(\mathbf{q}, \omega)=i \int_{0}^{+\infty} d t e^{i z t}\left\langle\left[X_{\mathbf{q}}(t), X_{-\mathbf{q}}(0)\right]\right\rangle,
$$

where $z=\omega+i \eta$, the angle brackets denote a canonical ensemble thermal average and the commutator plays a central role in the linear response theory (or Kubo) formulation of transport.

The two quantities are related by the fluctuationdissipation relation,

$$
\chi^{\prime \prime}(\mathbf{q}, \omega)=\frac{1-e^{-\beta \omega}}{2} S(\mathbf{q}, \omega)
$$

where $\beta=1 / k_{B} T$ is the inverse temperature. Note that $S(\mathbf{q}, \omega)$ satisfies the symmetry relation $S(-\mathbf{q},-\omega)=$ $e^{-\beta \omega} S(\mathbf{q}, \omega)$ while the sum-rule,

$$
\frac{1}{2 \pi} \int_{-\infty}^{+\infty} d \omega S(\mathbf{q}, \omega)=\left\langle X_{\mathbf{q}} X_{-\mathbf{q}}\right\rangle,
$$

makes it natural to consider the normalised to a unit area correlation function,

$$
\hat{S}(\mathbf{q}, \omega) \equiv \frac{S(\mathbf{q}, \omega)}{\left\langle X_{\mathbf{q}} X_{-\mathbf{q}}\right\rangle} .
$$

We have presented the dynamical correlation functions in the canonical ensemble and now we will establish their form in the microcanonical one. Starting from equation (8) and employing solely the idea that our distribution has a restricted energy $\lambda$ we can generate a correlation function $s(\mathbf{q}, \omega)$ in the microcanonical ensemble,

$$
s(\mathbf{q}, \omega)=\int_{-\infty}^{+\infty} d t e^{i \omega t} \sum_{m}\left\langle X_{\mathbf{q}} \mid m\right\rangle\left\langle m \mid X_{-\mathbf{q}}\right\rangle e^{i\left(\lambda-\epsilon_{m}\right) t}
$$

Here, we have used the relation,

$$
\langle O U(H)\rangle \mapsto\langle O\rangle U(\lambda)
$$

$(U(H)$ a function of $H)$ and a decomposition using the eigenbasis $|m\rangle$. The expression (13) integrates to provide,

$$
s(\mathbf{q}, \omega)=2 \pi \sum_{m}\left\langle X_{\mathbf{q}} \mid m\right\rangle\left\langle m \mid X_{-\mathbf{q}}\right\rangle \delta\left(\omega+\lambda-\epsilon_{m}\right),
$$


that can be re-represented as the basic correlation in the microcanonical ensemble,

$$
s(\mathbf{q}, \omega)=-2 \lim _{\eta \mapsto 0} \Im\left\langle X_{\mathbf{q}}[z-H+\lambda]^{-1} X_{-\mathbf{q}}\right\rangle .
$$

Notice that this expression is exact in the zero temperature limit where the expectation value is to be taken over the ground state wavefunction.

Now let us imagine that we could find a single eigenstate at will, with an energy arbitrarily close (in the thermodynamic limit) to a target energy, $\lambda$ say. It is in principle straightforward then to determine

$$
s^{*}(\mathbf{q}, \omega)=-2 \lim _{\eta \mapsto 0} \Im\left\langle *\left|X_{\mathbf{q}}\left[z-H+\epsilon_{*}\right]^{-1} X_{-\mathbf{q}}\right| *\right\rangle,
$$

exactly as before, where $H|*\rangle=\epsilon_{*}|*\rangle$ is the known eigenstate with $\epsilon_{*} \mapsto \lambda$. If the microcanonical ensemble is equivalent to the canonical ensemble and if a single eigenstate is representative of the microcanonical one, then provided that $\lambda=\langle H\rangle$ for the desired temperature, we can expect that

$$
s^{*}(\mathbf{q}, \omega) \mapsto S(\mathbf{q}, \omega)
$$

in the thermodynamic limit. This amounts to the physical idea behind our calculations.

Furthermore, from (10), it follows that

$$
\chi^{\prime \prime}(\mathbf{q}, \omega)=\frac{1-e^{-\beta \omega}}{2} s(\mathbf{q}, \omega)
$$

and from the symmetry of $S(\mathbf{q}, \omega)$ in the canonical ensemble we can deduce that,

$$
\ln \frac{s(\mathbf{q}, \omega)}{s(-\mathbf{q},-\omega)} \mapsto \beta \omega
$$

this relation then provides an alternative, cross-checking technique for determining the temperature for a particular value of $\lambda$. Although we might like to believe that a single eigenstate corresponds to the microcanonical ensemble, based on a putative ergodicity assumption for the eigenstate, in practice it is not possible to find such an eigenstate. So we relax the eigenstate hypothesis and go back to a distribution of eigenstates close to the desired value $\lambda$. We simply use the formalism as though we had such an eigenstate.

\section{THE LANCZOS METHOD}

In principle we must construct a particular eigenstate with energy $\lambda$ that equals the canonical expectation value of the energy, $\langle H\rangle=\lambda$, at the desired temperature. In practice, we employ the well known Lanczos algorithm that is an efficient way of diagonalising large Hamiltonians using as variational subspace (truncated basis) the set of states,

$$
\left\{|0\rangle, H|0\rangle, \ldots, H^{M_{1}}|0\rangle\right\},
$$

where $|0\rangle$ is a (usually random) initial state and $M_{1}+1$ the number of Lanczos steps. To obtain an eigenstate close to energy $\lambda$ one might expect to use the closest eigenstate to $\lambda$ in the truncated basis, but this is totally incorrect. In practice, only the states at the edge of the spectrum converge and the other 'eigenstates' in the truncated subspace have the suggested energies but are usually far from eigenstates.

In order to apply the Lanczos method idea, one can simply push the energetic region of interest to the edge of the spectrum by choosing an appropriate new operator. One natural choice is to use,

$$
K \equiv(H-\lambda)^{2}
$$

which is positive definite and pushes the eigenstates with energy close to $\lambda$ towards the minimal, zero, eigenvalue of $K$. Another way to understand this technique is to consider expanding the ground state of $K$, that we will call $|\lambda\rangle$, as a probability distribution over eigenstates. Choosing $\lambda$ establishes the appropriate mean for this distribution but minimising $K$ corresponds to minimising the variance of the distribution, and consequently localising the distribution near $\lambda$.

One can perform a Lanczos calculation based upon the operator $K$ or, more efficiently, one can evaluate the operator $K$ (now a pentadiagonal matrix) in a previously constructed Lanczos basis using $H$ ("L-projection" method). Note that,

$$
\left\langle(H-\langle H\rangle)^{2}\right\rangle=\left\langle(H-\lambda)^{2}\right\rangle-(\langle H\rangle-\lambda)^{2} \geq 0,
$$

(the expectation value is over $|\lambda\rangle$ ) and so a small variance guarantees a narrow distribution of energies around $\lambda$.

In any Lanczos calculation the mathematical orthogonality between states becomes lost at some stage as numerical errors build up. In practice only the wellseparated converged states suffer from this disease and for us these states, which are at the edge of the spectrum, do not gain any significant weight in the correlation functions and so do not manifest in our results. The states at low frequency are all well behaved and maintain their orthogonality.

It is straightforward to implement these ideas numerically, with a 'double-Lanczos' calculation; the first run through a Lanczos procedure of $M_{1}$ steps is employing the operator $K$ starting from a random state and it is used to find the state $|\lambda\rangle$ which plays the role of the microcanonical distribution. The second run of $M_{2}$ steps 
through Lanczos is made using $X_{\mathbf{q}}|\lambda\rangle$ as the initial state and then the resulting tridiagonal matrix can be diagonalised to form the dynamical correlations directly or by employing the continued fractions method which is numerically more efficient but introduces a loss of resolution.

All the analysis so far has been subject to several caveats; firstly, that the microcanonical ensemble is equivalent to the canonical one in the thermodynamic limit and in the context of quantum dynamic correlations. Secondly, that a single eigenstate is equivalent to the microcanonical ensemble and thirdly that we can find such an eigenstate at will. The first two assumptions, as we have mentioned in the introduction, should be the focus of analytical studies as fundamental issues of nonequibrium statistical mechanics.

Regarding the third assumption, it is clearly problematic as it is well known that although the Lanczos method converges quite easily at the sparse edges of the spectrum, in the denser inner regions of the spectrum, of interest at finite temperature, it takes the Lanczos procedure an exponentially large number of iterations to converge. A many-body spectrum has an exponential number of states, e.g. for spin- $1 / 2$ the \# (States) $\sim 2^{N}$, and for a bounded Hamiltonian the eigenstates are compressed into an energy region that grows only linearly with system size. Although the low energy region maintains a sparse density of states, the eigenstates become exponentially close together in the area of interest and essentially become unattainable.

At first sight this appears an insurmountable difficulty, but in practice this issue allows the technique its success. The first Lanczos procedure provides a single quantum state $|\lambda\rangle$, that is not an eigenstate, but which when decomposed in an eigenstate basis, it is represented by a narrow distribution $\left|a_{n}\right|^{2}$ around $\lambda$;

$$
\begin{aligned}
& |\lambda\rangle=\sum_{n} a_{n}|n\rangle, \quad H|n\rangle=\epsilon_{n}|n\rangle \\
& \langle\lambda|H| \lambda\rangle=\lambda,
\end{aligned}
$$

gives for the expectation value of an operator $O$,

$$
\begin{aligned}
\langle\lambda|O| \lambda\rangle & =\sum_{n}\left|a_{n}\right|^{2}\langle n|O| n\rangle \\
& +\sum_{n \neq m} a_{m}^{*} a_{n}\langle m|O| n\rangle .
\end{aligned}
$$

This state, used in the evaluation of expectation values, acts as a statistical average over an energy window. It is important to notice, that by employing a single quantum state (not eigenstate) for evaluating an expectation value (as a substitute for a statistical average over a narrow energy window of eigenstates), we assume that the appearing off-diagonal terms (second term in eq. (25) cancel each other. This assumption can be justified (and numerically verified) by invoking a random phase decomposition of the used quantum state.

From this discussion we can expect two types of fluctuations in the obtained spectra; first, intrinsic fluctuations due to the finite size of the system, present even when a single eigenstate is used for the evaluation of the expectation value. Second, statistical fluctuations entering by the off-diagonal terms in eq. (25) due to the use of a single pure state that is not an eigenstate; this type of fluctuations can be reduced by averaging over orthogonal states $|\lambda\rangle$ (e.g. corresponding to different translational symmetry $k$ - subspaces as we will show below).

\section{CONVERGENCE OF PROJECTION}

In the following we present a test on the rate of convergence of the projection to a single quantum state with energy close to $\lambda$. Due to the innate complexity of an implicit scheme like Lanczos, we develop the theory of a simpler technique briefly to exhibit the ideas.

A rather simple method of numerically solving for the ground state is by an iterative sequence of applications of the scaled Hamiltonian. For us this amounts to iterative applications of the operator,

$$
P=1-\left(\frac{H-\lambda}{\mu}\right)^{2}
$$

where $\mu$ is chosen to be large enough so that $\mu^{2}>$ $\left(\epsilon_{n}-\lambda\right)^{2}$ for the full spectrum. Repeated applications of this operator exponentially suppresses all states except those for which $\epsilon_{n} \sim \lambda$ which remain unaffected. We can start out with a set of random states and then for $M$ applications of our operator we can build a distribution,

$$
\rho_{M}(H)=\sum_{\psi} P^{M}|\psi\rangle \theta[P]\langle\psi| P^{M}
$$

$(\theta(P)$ is the step function) and if we were to perform an average over an orthogonal basis, $|\psi\rangle$, then this would converge to,

$$
\rho_{M}(H) \mapsto \theta[P] P^{2 M}
$$

Elementary analysis provides: 


$$
\rho_{M}(H)=\left.\int_{i \infty}^{-i \infty} \frac{d \beta}{2 \pi i} \exp [\lambda \beta-\beta H](2 M) ! 2^{2 M} 2 \mu\left[\frac{1}{x} \frac{d}{d x}\right]^{2 M} \frac{\sinh x}{x}\right|_{x=\beta \mu} .
$$

In the limit that $M \mapsto \infty$ we find that,

$$
f(\beta) \mapsto \lambda \beta+\frac{\mu^{2} \beta^{2}}{2(4 M+3)}+O\left(\frac{1}{M^{2}}\right)
$$

and we converge to a narrow Gaussian probability distribution,

$$
\rho_{M}(H) \sim \exp \left[-\frac{(H-\lambda)^{2}}{2 \mu^{2}}(4 M+3)\right] .
$$

The width of this distribution is under our control,

$$
\left\langle(H-\lambda)^{2}\right\rangle \sim \frac{\mu^{2}}{4 M+3} \sim \frac{W^{2} N^{2}}{4 M+3},
$$

where $W$ is the natural energy scale for the model and we see that $M$ needs to scale with the square of the system size $N$ to maintain resolution.

The Lanczos method is clearly much more sophisticated and provides a much narrower distribution. We have examined the distribution obtained in a Lanczos calculation and we find that it is well represented by a Gaussian distribution with a variance controlled by the 'eigenvalue' of $K$ attained by the calculation. In practice this is about two orders of magnitude better in energy than the result obtained from the projection analysis (eq.(26) ), which however it is analytically controllable; indeed, we find that the Lanczos method scales as $\langle K\rangle \propto M_{1}{ }^{-2}$ so that the intrinsic resolution, $\sigma=\sqrt{\langle K\rangle}$, is inversely proportional to the number of iterations. The convergence properties of the three schemes we discussed are depicted in Figure 1 for a representative calculation of the study that we present in the next section.

The application of the technique should now be transparent; employing a single random state, or averaging over a sequence of orthogonal random states, one performs a first Lanczos calculation of $M_{1}$ steps to find the approximate ground state $|\lambda\rangle$ for the operator $K=(H-\lambda)^{2}$. The value of $\lambda$ must be pre-selected so that $\lambda=\langle H\rangle$ for the chosen temperature; several techniques are available for reliably determining this energy versus temperature relation as the Bethe Ansatz (for integrable systems), the finite temperature Lanczos (FTLM $)^{3}$, the Transfer Matrix Renormalization Group (TMRG) or Quantum Monte Carlo method. The degree of convergence can be measured using the eventual 'eigenvalue' of $K$; it plays the role of the variance of the chosen distribution and its square-root is an intrinsic energy resolution $\sigma$. This scale, $\sigma$, can never drop below the distance to the nearest eigenvalue. For a usual size system,

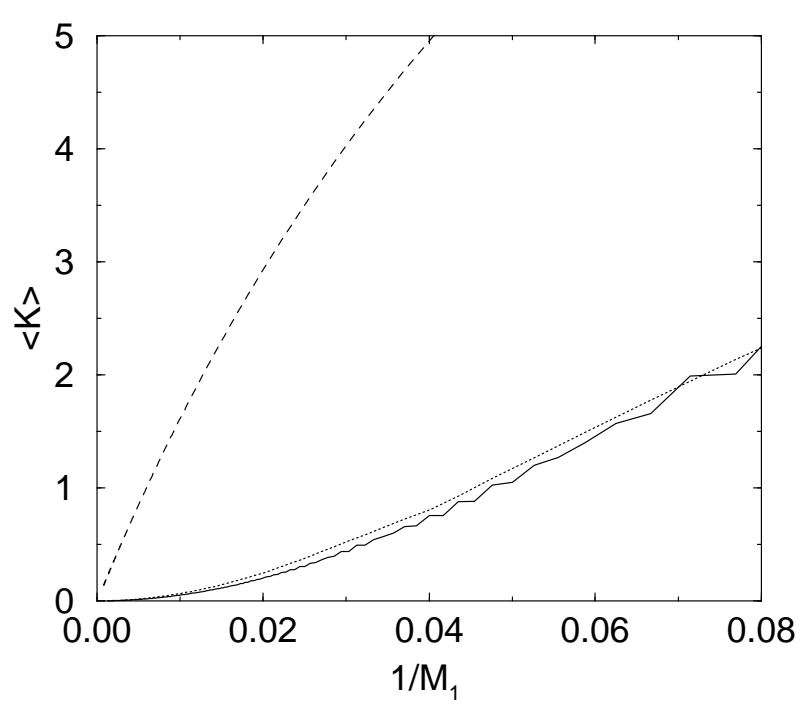

FIG. 1: Convergence properties of different Lanczos projection procedures: (i) dashed line, using eq.(26), (ii) dotted line, using $K=(H-\lambda)^{2}$, (iii) continuous line, "L-projection" (see text).

e.g. $N>16$, and temperature, this limit is unattainable but a resolution of $\sigma \sim 0.01(\langle K\rangle \sim 0.0001)$ is readily available with a thousand or so $M_{1}$ iterations.

Once one has found this state $|\lambda\rangle$, that plays the role of the state $|*\rangle$, a second Lanczos projection sequence is generated employing the state $X_{\mathbf{q}}|\lambda\rangle$ as the initial state. The resolution of the eventual result is controlled by the intrinsic dependence on the microcanonical ensemble and the degree of convergence measured by $\sigma$. This can be seen from relation (16) as the eigenstates over which the state $|\lambda\rangle$ is decomposed have a spread in energy $\sigma$ with respect to the reference energy $\lambda$. The resolution also depends on the convergence achieved in the 2nd Lanczos procedure where the number of iterations $M_{2}$ denotes the finite number of poles which are used to try to represent the dynamical correlations. At the sparse edges of the spectrum these poles denote the eigenvalues of the system but in the bulk of the spectrum, when grouped into bins of a given frequency width, they are fairly uniformly spread and offer a further natural energy resolution for the calculation.

More Lanczos steps provide more poles and a finer spectral 'grid' for the correlation functions, until the graininess of the real system is achieved. We have elected to use a few thousand poles in our calculations with very little improvement obtained by increasing this number as we shall see. The final resolution is self-imposed and 
is the $\eta$ of (16) which we choose to be of order of the spectral grid in order to smooth our calculations.

\section{APPLICATION ON THE SPIN-1/2 HEISENBERG MODEL}

We are now in a position to test our proposed technique and uncover its strengths and weaknesses. We have chosen to investigate the finite temperature dynamics of the prototype spin-1/2 Heisenberg model (equivalent to the fermionic " $t-V$ " model). This choice was dictated by its central role in low dimensional quantum magnetism; an exact solution of the thermodynamics and elementary excitations is known using the Bethe Ansatz method ${ }^{6}$, the spin dynamics probed by NMR is of current experimental interest ${ }^{7.8}$ and several numerical and analytical studied have been devoted to the study of finite temperature dynamic correlations 9.11,12,13,14. The Hamiltonian is given by,

$$
H=\sum_{l} h_{l}=J \sum_{l=1}^{N}\left(S_{l}^{x} S_{l+1}^{x}+S_{l}^{y} S_{l+1}^{y}+\Delta S_{l}^{z} S_{l+1}^{z}\right)
$$

where $S_{l}^{\alpha} \quad(\alpha=x, y, z)$ are spin-1/2 operators on site $l$ and we take $J$ as the unit of energy and frequency $(\hbar=$ $1)$.

In particular, we will look at the high temperature spin conductivity in the antiferromagnetic regime, $J, \Delta>0$, for which several studies exist and some exact results are known 13 . To discuss magnetic transport, we first define the relevant spin current, $j^{z}$, by the continuity equation of the corresponding local spin density $S_{l}^{z}$ (provided the total $S^{z}$ component is conserved),

$$
S^{z}=\sum_{l} S_{l}^{z}, \quad \frac{\partial S_{l}^{z}}{\partial t}+\nabla j_{l}^{z}=0
$$

Thus, we obtain for the spin current $j^{z}$, (that plays the role of the operator $X_{\mathbf{q}}$ ),

$$
j^{z}=\sum_{l} j_{l}^{z}=J \sum_{l}\left(S_{l}^{x} S_{l+1}^{y}-S_{l}^{y} S_{l+1}^{x}\right) .
$$

The real part of the "spin conductivity" $\sigma^{\prime}(\omega)$ (corresponding to the charge conductivity of the fermionic model) includes two parts, the Drude weight $D$ and the regular part $\sigma_{\text {reg }}(\omega)^{9,10}$,

$$
\sigma^{\prime}(\omega)=2 \pi D \delta(\omega)+\sigma_{r e g}(\omega) .
$$

The regular contribution is given by,

$\sigma_{r e g}(\omega)=\frac{1-e^{-\beta \omega}}{\omega} \frac{\pi}{N} \sum_{n \neq m} p_{n}|<n| j^{z}|m>|^{2} \delta\left(\omega-\omega_{m n}\right)$

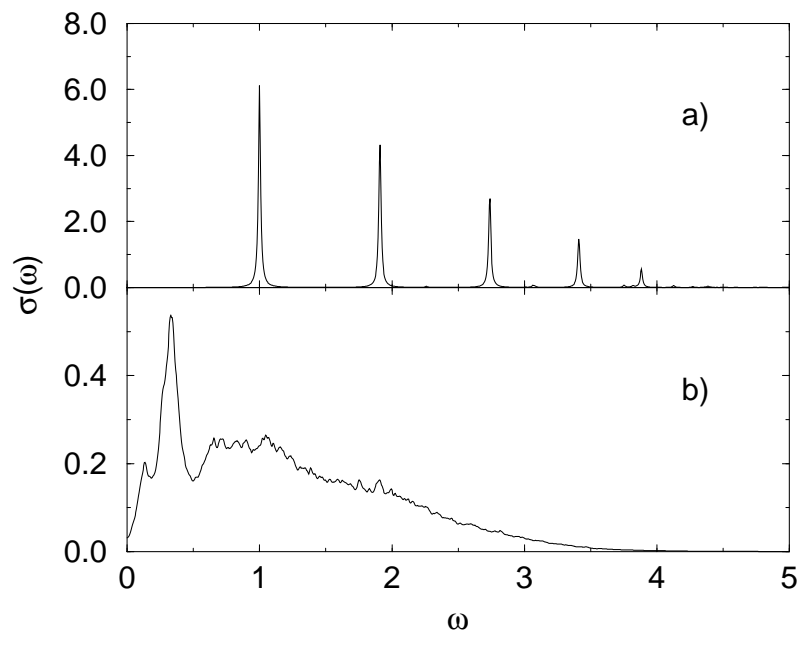

FIG. 2: Microcanonical calculations for $N=26, \Delta=2$, $\eta=0.02$; (a) $T=0$, (b) $\beta \rightarrow 0$.

where $p_{n}$ are the Boltzmann weights and $\omega_{m n}=\epsilon_{m}-\epsilon_{n}$.

To compare the presented data on the conductivity we normalize them using the well known optical sum-rule that in the $\beta \rightarrow 0$ limit takes the form,

$$
\int_{-\infty}^{+\infty} d \omega \sigma_{r e g}(\omega)+2 \pi D=\beta \frac{\pi}{N}\left\langle j^{2}\right\rangle
$$

The normalized conductivity, $\sigma(\omega)$, in this high temperature limit is given by,

$$
\sigma(\omega)=\frac{\sum_{n \neq m}|<n| j^{z}|m>|^{2} \delta\left(\omega-\omega_{m n}\right)}{\left\langle j^{z^{2}}\right\rangle},
$$

that can be calculated using our microcanonical ensemble procedure by,

$$
\sigma(\omega) \mapsto-\lim _{\eta \mapsto 0} \frac{\Im\left\langle\lambda\left|j^{z} \frac{1}{z-H+\lambda} j^{z}\right| \lambda\right\rangle}{\pi\left\langle\lambda\left|j^{z^{2}}\right| \lambda\right\rangle}
$$

In principle this expression includes also the contribution from the zero frequency Drude weight $\delta$-function, but in practice as the second Lanczos procedure cannot fully converge, the Drude peak appears as a low frequency contribution. As we will discuss below, sorting out this low frequency part, in general allows us to reliably extract the Drude weight value.

In general, we can employ the translational symmetry of the Hamiltonian and study spectra in a given pseudomomentum $k$ - subspace or average the results over different $k$ - subspaces; in the following we typically employ $M_{1}=1000$ and $M_{2}=4000$ Lanczos iterations at $\beta \rightarrow 0$ unless otherwise stated. In Figure 2 we compare a zero temperature $e^{2}$ with an infinite temperature $(\beta \rightarrow 0)$ calculation for a fairly large system in the $k=0$ subspace. 

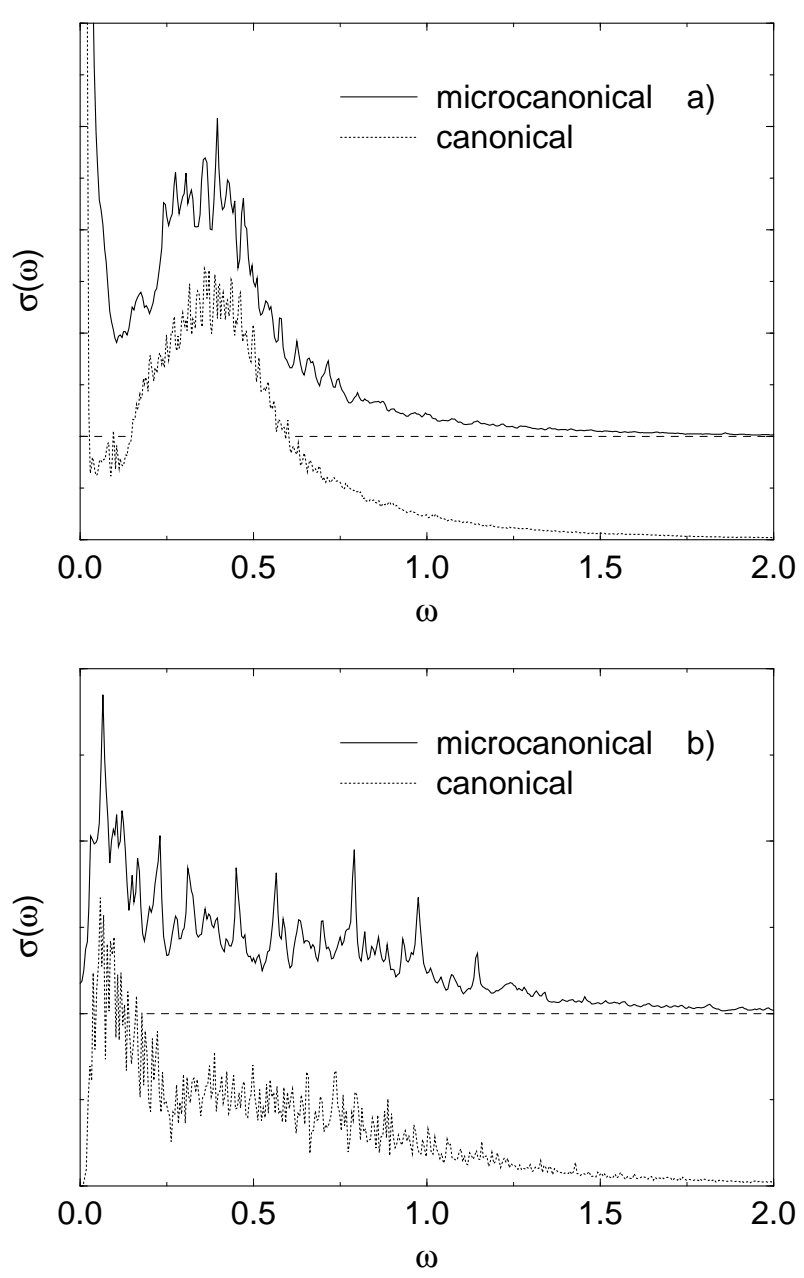

FIG. 3: Microcanonical versus Canonical calculations; (a) $N=20, \Delta=0.5, \eta=0.01$, (b) $N=18, \Delta=1, \eta=0.01$.

The zero temperature calculation finds a few poles with exact weights whereas the infinite temperature calculation provides a much smoother result.

There is clear structure in the infinite temperature result but also apparently some noise. To interpret this result we must consider the issue of the veracity of the microcanonical ensemble for such small systems namely the extent to which the microcanonical ensemble is equivalent to the canonical one.

In Figure 3 we present a comparison, extremely encouraging, of some microcanonical calculations with the analogous canonical ones. There is 'noise' in all calculations, the origin and magnitude of which we will now discuss. The canonical calculations are essentially a direct evaluation of expression (37), where we applied a "binning" procedure on the $\delta$-function weights over an energy scale of about 0.01 . The number of contributing matrix elements are of the order of the dimension $\mathcal{D}$ of the Hilbert space squared, $\mathcal{D}^{2}$, e.g. $10^{6}-10^{8} \delta-$ functions, with no continuity in the weights. The results are not smooth and the resulting intrinsic fluctuations are heavily smoothed by our binning procedure. In the microcanonical calculations we employ our scheme, further averaging over translational symmetry $k$-subspaces. Now, only of $O(\mathcal{D}) \delta$-functions are essentially contributing, multiplied by the number of states involved in the decomposition of the state $|\lambda\rangle$ (a few thousand depending on the convergence) and the number of $k$-subspaces. We could average over initial random states, but we find that this has only a small smoothing effect, because the underlying poles are at the same energies. Notice that the observed fluctuations are not associated with any of our different resolution processes which are much smaller than the observed scale of fluctuations; they are due to the finite size of our system and thus to the effective smaller number of matrix elements contributing to the construction of the spectra. This seemingly new problem associated with our technique turns out to be dominant for small system sizes; very soon however it becomes negligible as larger systems are achieved, specially considering that the dimension of Hilbert space grows exponentially fast with the system size $N$.

In order to assess these fluctuations and simultaneously the role of our smoothing parameter $\eta$, we performed some basic calculations involving only a single $k$-subspace state $|\lambda\rangle$. In Figure 4 we offer a comparison of calculations involving just the poles evaluated using the 2nd Lanczos procedure eigenstates against smoothed versions of the same data but employing the continued fraction technique.

The fluctuations clearly decay with system size with the final system being surprisingly smooth. The limitations of the smoothing process are clear, the sharper features are slightly washed out although the ease of assessing the data makes such a smoothing advisable. The weights for these microcanonical calculations are truly quite continuous in comparison to the intrinsic properties of the canonical calculation which is necessarily ragged. Obviously for our largest calculations we are nowhere near converged to the true spectrum which is a possible explanation for the observed continuity.

We can now fairly safely conclude that our technique is a viable way to calculate dynamical correlation functions at high temperature for the same systems accessible by the Lanczos method at $T=0$. By its very nature, the finite $T$ correlations are much smoother and more regular to interpret. Our technique introduces new statistical fluctuations which make small system sizes ragged but appear to leave large system sizes essentially unaffected.

Although we can now investigate finite temperature dynamic correlations using the Lanczos method, we are still restricted to $N \sim 30$ for a spin- $1 / 2$ system. The key to making useful physical deductions is the procedure of finite-size scaling, the attempt to deduce the properties of the infinite size system using assumed properties of the size, $N$, dependence. This method has been widely and succesfully applied in the evaluation of ground state energies or gap values using data provided by the exact diagonalization, Lanczos or Density Matrix Renormal- 

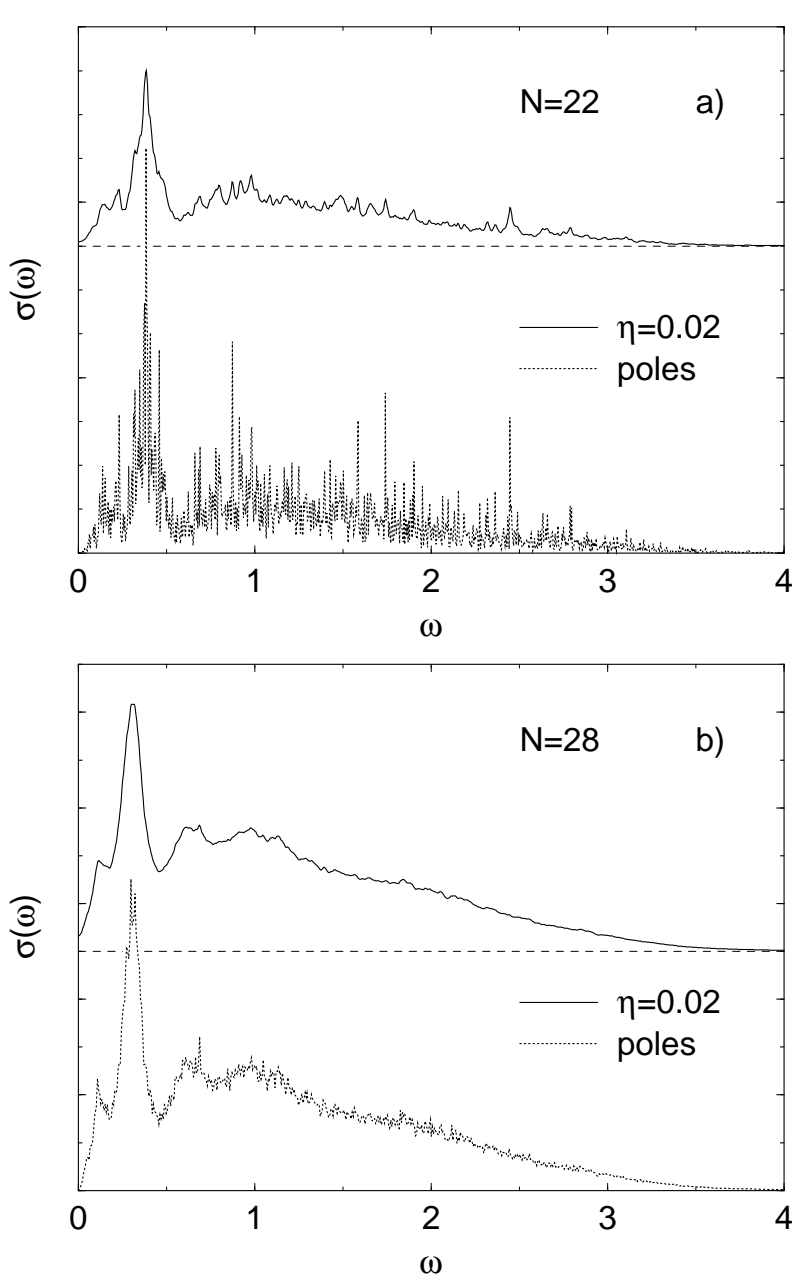

FIG. 4: Microcanonical finite-size effects for $\Delta=2$; (a) $\mathrm{N}=22$, (b) $\mathrm{N}=28$.

ization Group technique. But to extract information on finite temperature dynamic correlations one would need to know the form of the curves before fitting and scaling could take place mathematically. As it is clear from Figure 5 this might be a challenging task considering the statistical fluctuations inherent in the spectra ${ }^{15}$; however, from ongoing studies on other systems using this method, we find that the behavior of the spectra might greatly depend on the model Hamiltonian and correlations under study (e.g. it is far more structurless for energy current dynamic correlations). Note that the high frequency behavior is generally rather weakly size dependent while the low frequency one is the most subtle to determine. The last however is the most physically interesting as it determines, for instance, the diffusive or ballistic behavior of the conductivity.

The basic properties of the $\beta \rightarrow 0$ current-current correlations are now available and so we provide in Figure 6 a few examples of the frequency dependence of the conductivity at $\beta \rightarrow 0$ as a function of $\Delta$.

Although we have devoted most of our effort to infi-

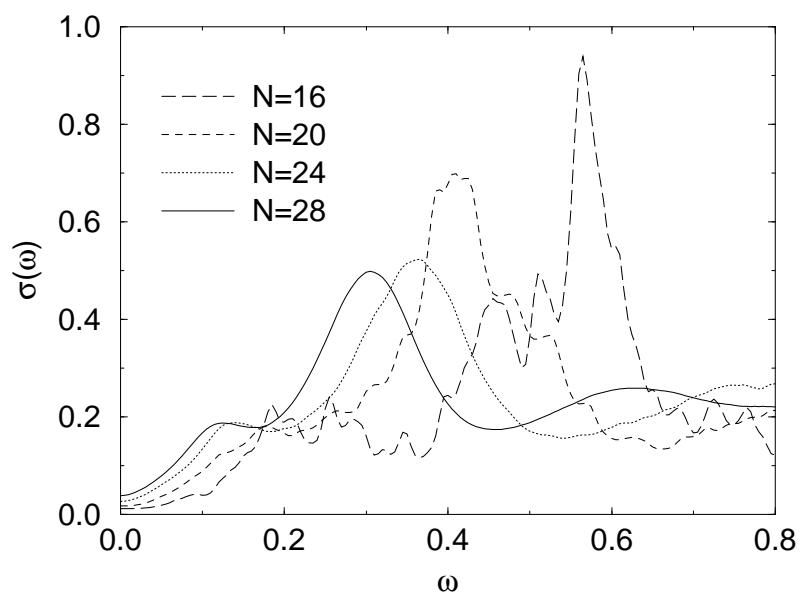

FIG. 5: Finite-size scaling for $\Delta=2$
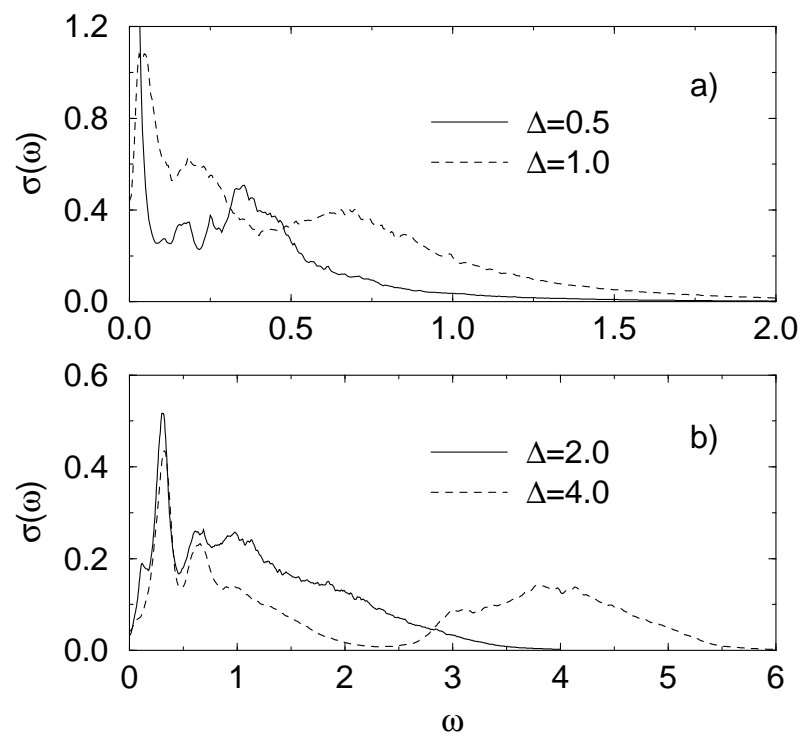

FIG. 6: Microcanonical ensemble evaluation of the normalized conductivity $\sigma(\omega)$ for $\beta \rightarrow 0, \quad N=28$; (a) $\Delta=0.5,1.0$, (b) $\Delta=2.0,4.0$

nite temperature $(\beta \rightarrow 0)$, our technique is valid at essentially any temperature (provided that we remain at a dense region of the spectrum). Analysing the pure Heisenberg model, we look at a couple of finite temperature $k$-averaged calculations in Figure 7 The temperature has been deduced from a least-squares fit of the quantity,

$$
\ln \frac{s(\omega)}{s(-\omega)} \sim \alpha+\beta_{\text {micro }} \omega
$$

to a linear Ansatz, and although the statistical fluctuations are compounded, an almost vanishing intercept and a clear slope indicate the feasibility of the strategy. The obtained $\beta_{\text {micro }}$ values compare favorably with those corresponding to the canonical ensemble in the 

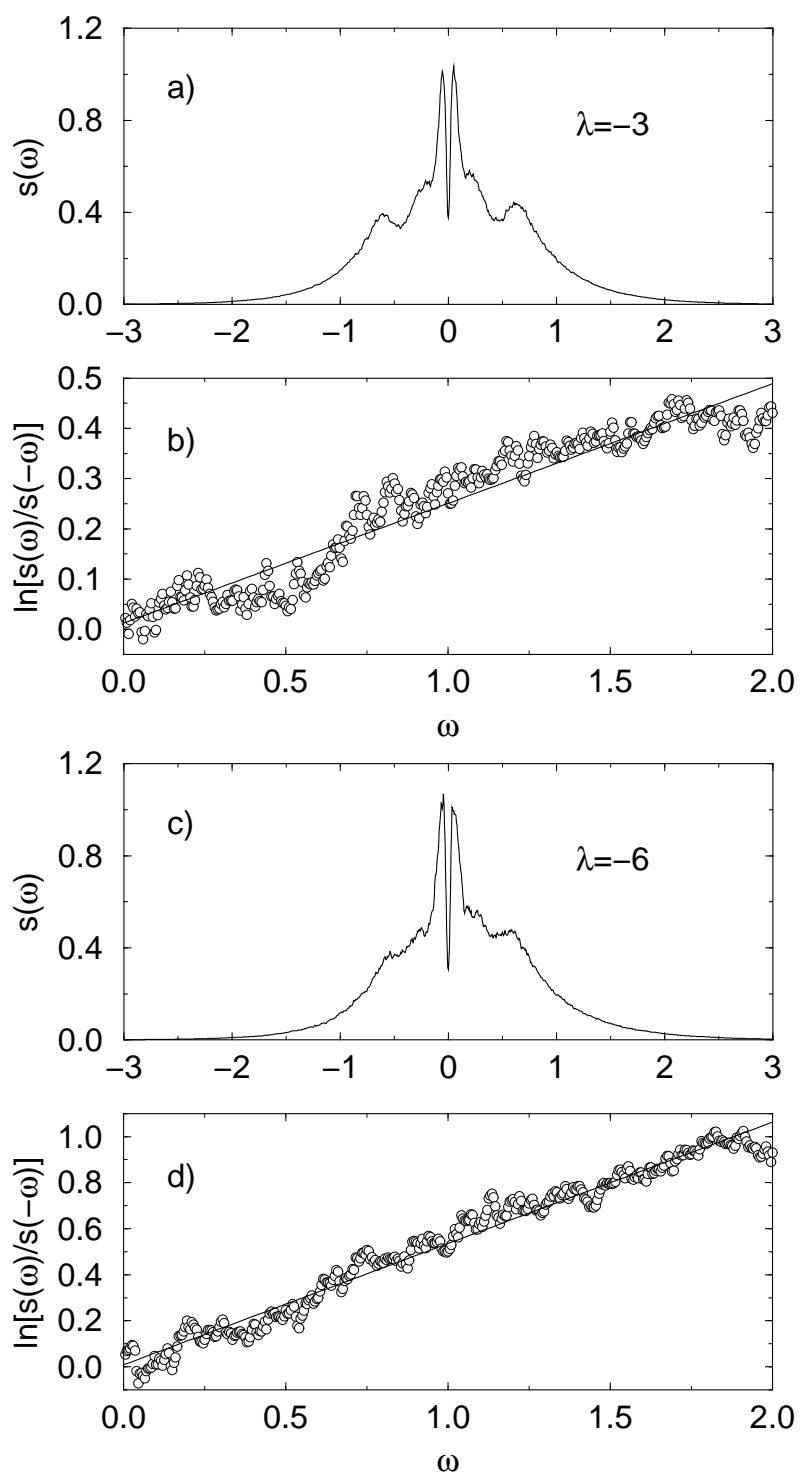

FIG. 7: Finite temperature calculations for $N=24, \Delta=1$, $\eta=0.01$; (a) $s(\omega), \lambda=-3$, (b) Temperature fit $\beta_{\text {micro }} \simeq 0.14$, (c) $s(\omega), \lambda=-6$, (d) Temperature fit $\beta_{\text {micro }} \simeq 0.28$.

thermodynamic limit, evaluated using $\lambda=<H>_{\beta}$; for $\lambda=-3, \beta_{\text {micro }} \sim 0.14$ vs. $\beta_{\text {canonical }} \sim 0.15$, for $\lambda=-6$, $\beta_{\text {micro }} \sim 0.28$ vs. $\beta_{\text {canonical }} \sim 0.3$.

Although we have compared numerical evaluation of dynamic correlations obtained by a canonical and microcanonical method, we have yet to compare with an exact solution. Recently even non-zero temperature dynamical correlations have become partially accessible, with a calculation of the Drude weight for the $0<\Delta<1$ Heisenberg model at finite temperature ${ }^{13}$. In particular, the Drude weight in the $\beta \rightarrow 0$ limit is given analytically 17 by,

$$
D / \beta=\frac{1}{2} \frac{(\pi / \nu-0.5 \sin (2 \pi / \nu))}{8 \pi / \nu}, \quad \Delta=\cos (\pi / \nu)
$$

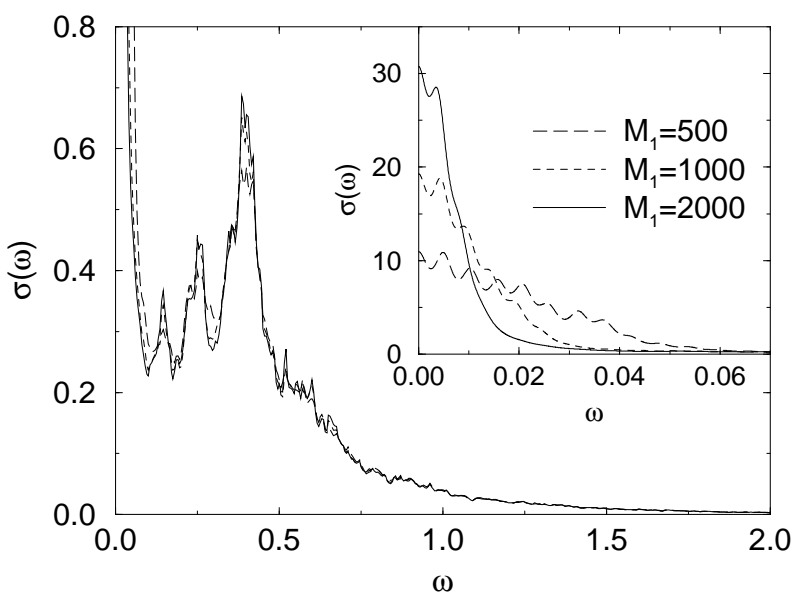

FIG. 8: A comparison of three 'microcanonical' distributions $\langle K\rangle=0.002\left(M_{1}=500\right), 0.0005\left(M_{1}=1000\right), 0.0012\left(M_{1}=\right.$ 2000), for $N=26$ and $\Delta=0.5$; inset, low frequency range.

The Drude weight, strictly speaking, is defined as the weight of a zero frequency $\delta$-function, eq.(36); it is a particularity of the Heisenberg model that it appears as a narrow peak at low frequencies, of the order of the inverse lattice size $\frac{16}{}$, in contrast to the fermionic " $t-\mathrm{V}$ " version where it is accounted for only by the diagonal energy elements $(\omega=0)$.

In extracting the Drude weight by the above described procedure we must take into account the problem caused by the intrinsic resolution of our calculations, which is of order $\sigma=\sqrt{\langle K\rangle}$. Although our chosen resolution of $\sigma \sim 0.01$ is almost invisible for the smooth background, for the Drude weight the resolution is essentially limited by that of our 'microcanonical' distribution, viz $\sigma$. An example of these ideas is provided in Figure 8 from which it is clear that the Drude peak is the only contribution for which the change in resolution is relevant. These calculations involve a single state and are much improved by $k$-averaging, also the energy window is so small that the individual poles in the 2nd Lanczos procedure are visible and have been smoothed out with an $\eta=0.005$ which adds to the observed resolution. In the inset, the scale of the conductivity clearly signals a low frequency peak (notice the difference in scale between Figure 8 and its inset); still in order to extract the Drude weight from the smooth background, we must integrate the peak up to at least as far as it is resolved and that necessitates the inclusion of some of the background. We have elected to err on the side of inclusion and tend to integrate past where the Drude peak appears to become small.

In Figure 9 we offer a comparison of the analytical and numerically extracted Drude weights in the $\beta \rightarrow 0$ limit. The quantitative agreement is reasonably satisfactory, becoming rather poor near $\Delta \sim 1$ because of our technique for extracting the Drude weight; due to the finite resolution of our calculation we need to sample a finite width around $\omega=0$. For the case $\Delta=1$ there is 


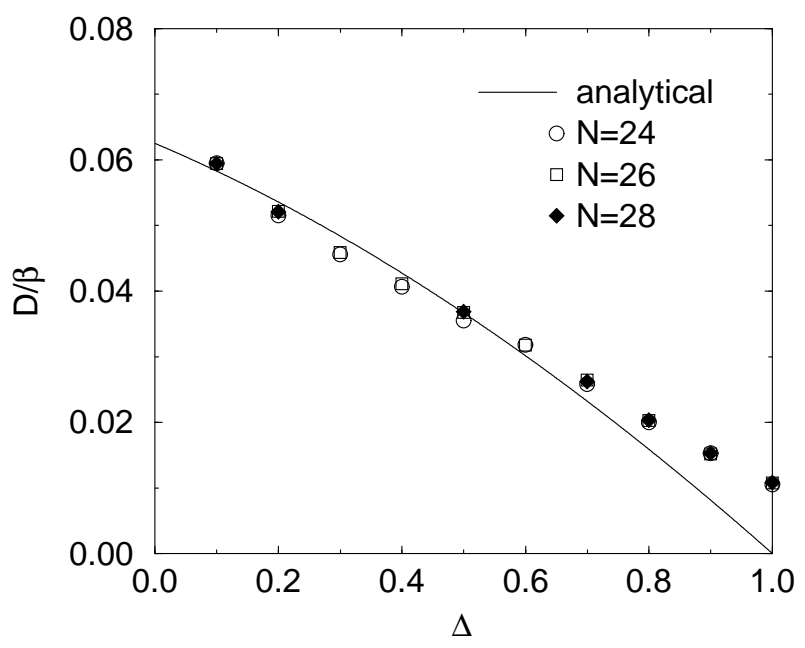

FIG. 9: Comparison of $\beta \rightarrow 0$ Drude weight, $D / \beta$; numerical evaluation (points) vs analytical expression eq. (42) (continuous line).

no Drude weight but there does appear to be a powerlaw like divergence which we pick up in our finite window leading to the observed corrupted behaviour.

\section{DISCUSSION}

Our investigation appears to validate the use of the Lanczos algorithm to analyse finite temperature dynamical properties of strongly correlated systems; the crucial step is to employ the microcanonical ensemble, which essentially allows the thermodynamic average to be replaced by an elementary expectation value. All the simplicity of the zero temperature formalism can then be taken over to the finite temperature calculation. The comparison of canonical with microcanonical procedures indicates that the thermodynamic limit is reached with quite modest system sizes and consequently there appears to be little systematic error coming from our choice of ensemble. There are intrinsic statistical fluctuations in our calculations but these are severely curtailed by increasing the system size and are an implicit difficulty with canonical calculations too. We believe that we can calculate the high enough temperature dynamical correlations for a finite system with an excellent tolerance.

The statistical fluctuations in our results require to be controlled if an error analysis is to be contemplated. Al- though we have not got analytical control, we do have experience at various approaches to reducing the statistical fluctuations. The crucial point is that, when taking a statistical average, one should use "orthogonal" states (| $\lambda\rangle$ 's decomposed into different sets of eigenstates $|n\rangle$ ). Averaging over random starting vectors in the same subspace is not very effective, even if they are originally orthogonal, because the resulting distribution involves the same states and consequently an overlap. Performing a $k$ - average, over translational symmetry subspaces, is an excellent procedure, since the states are automatically orthogonal and intellectually one is reverting back towards the real physical statistical average. Another possibility is to use several of the eigenstates of the first Lanczos procedure; although the orthogonality is guaranteed, there is an induced loss in resolution due to the larger $\sigma$ 's of the higher Lanczos states. A final possibility is to employ the parameter $\lambda$, where the average over different $\lambda$ 's must be limited within a window that corresponds to the energy fluctuations at the studied temperature in the given size system. Providing that the $\lambda$ 's are further apart than the chosen $\sigma$, the orthogonality is essentially guaranteed.

Although we believe we have access to the temperature behaviour of finite-size systems, this does not give immediate access to the dynamics in the thermodynamic limit because finite-size scaling must be performed; Figure 6 exhibits clear peaks of unknown form, plausible 'cusps' and regions where the correlations vanish. Unless we can guess or deduce the form of these structures, finite-size scaling appears problematic. We should note however from our experience, that not all models and dynamic correlations exhibit so involved spectra; in forthcoming works we will present analysis of charge/spin/energy current correlations for other (non-) integrable systems of current interest (higher spin, ladder models) where the obtained spectra are far more structrurless. Finally, besides the finite frequency behavior, our method allows the reliable study of scalar quantities as the Drude weight.

\section{Acknowledgments}

Part of this work was done during visits of (P.P.) and (M.L.) at IRRMA as academic guests of EPFL. J.K. and X.Z acknowledge support by the Swiss National Foundation, the University of Fribourg and the University of Neuchâtel.
1 R. Haydock, V. Heine and M.J. Kelly, J. Phys. C5, 2845 (1972).

2 E.R. Cagliano and C.A. Balseiro, Phys. Rev. Lett. 59, 2999 (1987).

3 J. Jaklič, P. Prelovšek, Phys. Rev. B49, 5065 (1994); Adv.
Phys. 19, 1 (2000).

${ }^{4}$ L.D. Landau and E.M. Lifshitz, "Course of Theoretical Physics: Statistical Mechanics", 5, 377 (footnote), Pergamon Press, London - Paris (1959).

5 J.L. Lebowitz, J.K. Percus and L. Verlet, Phys. Rev. 153, 
250 (1967).

6 "Quantum Inverse Scattering Method and Correlation Functions", V.E. Korepin, N.M. Bogoliubov and A.G. Izergin, Cambridge Univ. Press (1993).

7 M. Takigawa et al., Phys. Rev. Lett. 76, 4612 (1996).

${ }^{8}$ K.R. Thurber et al., Phys. Rev. Lett. 87, 247202 (2001).

9 X. Zotos and P. Prelovšek, Phys. Rev. B53, 983 (1996).

10 X. Zotos, F. Naef and P. Prelovšek, Phys. Rev. B55 11029 (1997).

11 K. Fabricius and B.M. McCoy, Phys. Rev. B57, 8340 (1998) and references therein.

12 B.N. Narozhny, A.J. Millis and N. Andrei, Phys. Rev. B58, 2921 (1998).
13 X. Zotos, Phys. Rev. Lett. 82, 1764 (1999).

14 J. V. Alvarez and C. Gros, Phys. Rev. Lett. 88, 077203 (2002).

15 The low frequency behavior of the spin-1/2 Heisenberg model, in particular the finite-size dependence as extracted from such a microcanonical study, will be adressed in a forthcoming publication.

16 F. Naef and X. Zotos, J. Phys. C. 10, L183 (1998); F. Naef, $\mathrm{Ph}$. D. thesis no.2127, EPF-Lausanne (2000).

17 A. Klümper, private communication.

18 S. Fujimoto and N. Kawakami, J. Phys. A. 31, 465 (1998). 